\title{
Relativistic calculations of two-color two-photon K-shell ionization
}

\author{
J. Fan ${ }^{1,2,3, a} \mathbb{D}$, J. Hofbrucker ${ }^{3,4}$, A. V. Volotka ${ }^{5}$, and S. Fritzsche ${ }^{2,3,4}$ \\ 1 Abbe School of Photonics, Albert-Einstein-Straße 6, 07745 Jena, Germany \\ 2 Theoretisch-Physikalisches Institut, Friedrich-Schiller-Universität Jena, Max-Wien-Platz 1, 07743 Jena, Germany \\ 3 Helmholtz-Institut Jena, Fröbelstieg 3, 07743 Jena, Germany \\ ${ }^{4}$ GSI Helmholtzzentrum für Schwerionenforschung GmbH, Planckstrasse 1, 64291 Darmstadt, Germany \\ 5 School of Physics and Engineering, ITMO University, Kronverkskiy pr. 49, St. Petersburg, Russia 197101
}

Received 7 September 2021 / Accepted 19 December 2021 / Published online 4 February 2022 (C) The Author(s) 2022

\begin{abstract}
We investigate the two-color two-photon $K$-shell ionization of neutral atoms based on the relativistic second-order perturbation theory and independent particle approximation. Analytical expressions for the relativistic and nonrelativistic total cross sections are derived in terms of radial transition amplitudes and Stokes parameters. Particular attention is paid especially to how the two-photon ionization total cross section depends on the energy sharing and polarization of the two incident photons. We construct the nonrelativistic expressions of cross section ratios for different polarization combinations of the two incident photons. The numerical results of total cross section and cross section ratios show that the energy sharing of the two incident photons plays an essential role in two-photon $K$-shell ionization. Particularly, if the energies of the two incident photons are identical, the total cross section and cross section ratios will reach the minimum or maximum value. Moreover, due to the strong screening effects, we find strong deviations of the cross section ratios near the two-photon ionization threshold of the Ne atom.
\end{abstract}

\section{Introduction}

The advent of high-intensity x-ray free-electron lasers (XFELs) has opened frontiers to study nonlinear ionization processes, such as the two-photon ionization (TPI) [1-3]. The TPI is one of the most fundamental nonlinear phenomena in the light-matter interaction process, in which an atom absorbs two photons and emits a photoelectron. Current XFEL facilities can produce pulses with $\mathrm{keV}$ photon energies [4-6] and offer the possibility to explore the inner shell TPI process. One of the first experiments has been performed for direct two-photon ionization of helium-like ion $\mathrm{Ne}^{8+}[7]$. Recently, studies were conducted for the two-photon $K$-shell ionization of solid targets, such as Ge [8], solid Zr [9], as well as metallic $\mathrm{Cu}[10,11]$.

From a theoretical point of view, the perturbative nonrelativistic framework has been employed in the calculations of total two-photon $K$-shell ionization cross section of many-electron systems in Refs. [12-15]. Further studies of TPI cross section have been conducted within the full relativistic framework for ionization of hydrogen-like atoms $[16,17]$, and later for neutral atoms $[18,19]$. Although the studies of two-photon $K$-shell ionization of atoms by monochromatic light are well established, the TPI process with two nondegenerate incident photons (bichromatic light) was paid less attention in

\footnotetext{
${ }^{\mathrm{a}}$ e-mail: jiahao.fan@uni-jena.de (corresponding author)
}

the past, which should be considered in the context of this work. Nevertheless, let us first discuss the techniques for the realization of the above-mentioned experimental scenario.

Various schemes for generation of two-color beams at XFEL facilities have been proposed [20-25], for instance utilizing variable-gap undulators [21], or using a single monochromatizing crystal as demonstrated in Ref. [22]. Recently, the methods by using a double-slotted foil on a chirped beam [23] or sextupole magnet [24] have been performed. In contrast to single-photon ionization, the total cross section of TPI shows a strong dependence not only on the energies of the photons but also on their polarizations. However, so far, the investigation has been focused on two equally polarized photons. Experimentally, the polarization control at XFEL facilities can be achieved either by various undulator configurations or by periodic temporal modulation [26$32]$. such as by employing crossed planar undulators, an arbitrary photon polarization can be generated as demonstrated in Refs. [26,28,31]. These examples hint that the techniques of generating two beams with tunable energies and polarizations will be available in the near future.

In this context, an important step is to investigate how the total cross section of two-color two-photon $K$ shell ionization depends on energy sharing and polarization of the two incident photons. Therefore, in Sect. 2 we firstly employ the relativistic second-order pertur- 
bation theory based on the Dirac equation and independent particle approximation to derive the total TPI cross section. We then apply the nonrelativistic limit to obtain simple expressions of cross section ratios for different polarization combinations. In Sect. 3, numerical computations have been carried out for two-color two-photon $K$-shell ionization of neutral $\mathrm{Ne}$ and $\mathrm{Ge}$ atoms. The total cross section and cross section ratios have been calculated as a function of energy sharing between the two incident photons. The results for the total cross section indicate that the minimum values occur when the energies of the two incident photons are identical. Similar behaviors occur in the results of cross section ratios as well. Besides, by comparing the analytical and numerical work of cross section ratios, we confirm the boundary values of cross section ratios for different polarization combinations. Finally, a summary is given in Sect. 4. For convenience, relativistic units $(\hbar=c=m=1$ ) are used throughout the paper, unless stated otherwise.

\section{Theoretical background}

In order to simplify the TPI process from many-electron system to single-electron system, we employ the independent particle approximation. We assume that an initial bound active electron of the $K$-shell $\left|n_{a} \kappa_{a} m_{a}\right\rangle$ interacts with the two incident photons. Here, $n_{a}$ is the principle quantum number, $\kappa_{a}$ is the Dirac quantum number, and $m_{a}$ is the projection of total angular momentum of the initial bound active electron. The Dirac quantum number $\kappa$ is defined by the total and orbital angular momenta $j$ and $l$ as $\kappa=\mp\left(j+\frac{1}{2}\right)$ for $j=l \pm \frac{1}{2}$. In addition, we use $\kappa_{\nu}$ and $\kappa$ to represent the Dirac quantum number of intermediate virtual state and continuum state throughout the paper. Since in this work, we investigate the scenario that the electron interacts with two photons $\gamma(\boldsymbol{k}, \boldsymbol{\epsilon})$ with different wave vectors $\boldsymbol{k}$ and polarization $\boldsymbol{\epsilon}$. We also assume the two incident photons propagate along the quantization axis $\left(\hat{\boldsymbol{k}}_{1}=\hat{\boldsymbol{k}}_{2}=\hat{\boldsymbol{k}}\right)$. The screening effect of all other inactive electrons is accounted for with a screening potential included in the Hamiltonian of the Dirac equation [18]. The two-color two-photon ionization can be presented as follows

$$
\left|n_{a} \kappa_{a} m_{a}\right\rangle+\gamma_{1}\left(\boldsymbol{k}_{1}, \boldsymbol{\epsilon}_{1}\right)+\gamma_{2}\left(\boldsymbol{k}_{2}, \boldsymbol{\epsilon}_{2}\right) \rightarrow\left|\boldsymbol{p}_{e} m_{e}\right\rangle
$$

here, we consider the ionization of $K$-shell electron, the above quantum numbers take the values $n_{a}=1$, $\kappa_{a}=-1$ and $m_{a}= \pm \frac{1}{2}$. After the interaction, a photoelectron is emitted into a continuum state $\left|\boldsymbol{p}_{e} m_{e}\right\rangle$ with well-defined asymptotic momentum $\boldsymbol{p}_{e}$ and spin projection $m_{e}$. In the following subsections, we apply the second-order relativistic perturbation theory as well as photon helicity density matrix, in order to derive the total cross section for the two-color two-photon $K$ shell ionization of an atom with a single active electron.
In the last subsection, we investigate the cross section ratios for different polarization combinations of $\boldsymbol{\epsilon}_{1}$ and $\boldsymbol{\epsilon}_{2}$ of the two incident photons.

\subsection{Evaluation of transition amplitudes}

To derive the total cross section of two-color TPI, one first has to obtain the transition amplitude. Generally, the total transition amplitude of the two-photon ionization consists of two terms. The first term corresponds to the ionization process where the first photon with energy $\omega_{1}$ excites the electron from the initial state into an intermediate virtual state, and the second incident photon with energy $\omega_{2}$ ionizes the electron from an intermediate virtual state into the continuum state. The second term arises from the process where the photon with energy $\omega_{2}$ is absorbed first and then the photon with energy $\omega_{1}$ promotes the active electron to continuum state. According to the second-order perturbation theory based on the Dirac equation, the first term of the transition amplitude can be written as

$$
\begin{aligned}
& M_{\boldsymbol{p}_{e} m_{e}}^{\lambda_{1} \lambda_{2}}\left(\omega_{1}, \omega_{2}\right) \\
& =\oiint_{\nu} \frac{\left\langle\boldsymbol{p}_{e} m_{e}\left|\boldsymbol{\alpha} \cdot \boldsymbol{A}_{\lambda_{2}}\left(\omega_{2}\right)\right| \nu\right\rangle\left\langle\nu\left|\boldsymbol{\alpha} \cdot \boldsymbol{A}_{\lambda_{1}}\left(\omega_{1}\right)\right| n_{a} \kappa_{a} m_{a}\right\rangle}{E_{n_{a} \kappa_{a}}+\omega_{1}-E_{n_{\nu} \kappa_{\nu}}} .
\end{aligned}
$$

Herein, $\boldsymbol{\alpha}$ denotes the vector Dirac matrices and $\mathbf{A}_{\lambda}(\omega)$ represents the photon field, the dot product of $\boldsymbol{\alpha} \cdot \boldsymbol{A}_{\lambda}(\omega)$ represents the electron-photon interaction operator in the Coulomb gauge. The summation is carried out over the complete spectrum of the intermediate electron states $|\nu\rangle . E_{n_{a} \kappa_{a}}$ and $E_{n_{\nu} \kappa_{\nu}}$ represent the binding energies of initial and intermediate electron states, respectively. For convenience, the expression for the second term of transition amplitude $M_{p_{e} m_{e}}^{\lambda_{2} \lambda_{1}}\left(\omega_{2}, \omega_{1}\right)$ can be easily obtained by the replacement $\omega_{1} \longleftrightarrow \omega_{2}$ and $\lambda_{1} \longleftrightarrow \lambda_{2}$. The transition amplitude for two-color TPI is given then by the sum $M_{p_{e} m_{e}}^{\lambda_{1} \lambda_{2}}=M_{p_{e} m_{e}}^{\lambda_{1} \lambda_{2}}\left(\omega_{1}, \omega_{2}\right)+$ $M_{p_{e} m_{e}}^{\lambda_{2} \lambda_{1}}\left(\omega_{2}, \omega_{1}\right)$. The single-electron transition amplitudes can be expanded in terms of spherical tensors by using the multipole decomposition of the photon field [33]

$$
\mathbf{A}_{\lambda}(\omega)=4 \pi \sum_{L M p} i^{L-p}\left[\hat{\boldsymbol{\epsilon}}_{\lambda} \cdot \boldsymbol{Y}_{L M}^{(p) *}(\hat{\boldsymbol{k}})\right] \boldsymbol{a}_{L M}^{(p)}(\mathbf{r})
$$

where $\boldsymbol{Y}_{L M}^{(p)}$ is vector spherical harmonic, the vector functions $\boldsymbol{a}_{L M}^{(p)}(\mathbf{r})$ refer to multipole potentials, where $L$ represents the order of multipole, and the index $p$ describes the electric $(p=1)$ and magnetic $(p=0)$ components of the electromagnetic field. If we choose the photon propagation direction as the quantization $\hat{\boldsymbol{k}} \| \boldsymbol{e}_{z}$, the scalar product of the polarization vector with the vector spherical harmonics becomes 


$$
\hat{\boldsymbol{\epsilon}}_{\lambda} \cdot \boldsymbol{Y}_{L M}^{(p) *}(\hat{\boldsymbol{k}})=\sqrt{\frac{[L]}{8 \pi}}(-\lambda)^{p} \delta_{\lambda M}
$$

Apart from the radiation field, we also need to expand the wave function of the outgoing electron into its partial waves $[34]$

$$
\begin{aligned}
& \left|\boldsymbol{p}_{e} m_{e}\right\rangle=\frac{1}{\sqrt{E_{e}\left|\boldsymbol{p}_{e}\right|}} \sum_{j m_{j}} \sum_{l m_{l}} i^{l} e^{-i \Delta_{j l}}\left\langle l m_{l}, \frac{1}{2} m_{e} \mid j m_{j}\right\rangle \\
& \quad \times\left|E_{e} j l m_{j}\right\rangle Y_{l m_{l}}^{*}\left(\hat{\boldsymbol{p}}_{e}\right)
\end{aligned}
$$

where $E_{e}=\sqrt{\boldsymbol{p}_{e}^{2}+m^{2}}$ is the electron energy, $\Delta_{j l}$ is the phase factor, the bracket $\langle. ., . . \mid .$.$\rangle represents the$ Clebsch-Gordan coefficients, and $\left|E_{e} j l m_{j}\right\rangle$ stands for partial waves of the continuum electron. Note that the spherical harmonics $Y_{l m_{l}}^{*}\left(\hat{\boldsymbol{p}}_{e}\right)$ characterize the direction of emitted electron. The summation in the expression (4) runs over all total and orbital angular momentum quantum numbers $j$ and $l$ of the continuum electron. Furthermore, we carry out the angular integration of the $\hat{\boldsymbol{r}}$ vector of the transition amplitude and expand the expression (1) in terms of radial transition amplitude $U_{\kappa_{a} \kappa_{\nu} \kappa}^{p L L L^{\prime}}$. The the radial part of the transition amplitude $U_{\kappa_{a} \kappa_{\nu} \kappa}^{p L L p^{\prime} L^{\prime}}$ corresponds to the two-photon ionization channel: $\kappa_{a} \rightarrow \kappa_{\nu} \rightarrow \kappa$ and describes the interaction independently of the magnetic quantum numbers $m_{a}, m_{\nu}$, and $m_{j}$. An explicit expression of the radial integrals can be found in Eqs.(6.129) in Ref. [35]. By carrying out all above simplifications, the transition amplitude obeys the following equation

$$
\begin{aligned}
& M_{p_{e} m_{e}}^{\lambda_{1} \lambda_{2}}\left(\omega_{1}, \omega_{2}\right)=\frac{1}{2} \sum_{p_{1} L_{1}} \sum_{p_{2} L_{2}} \sum_{n_{\nu} \kappa_{\nu} m_{\nu}} i^{L_{1}-p_{1}+L_{2}-p_{2}} \\
& \quad \sqrt{L_{1}\left(L_{1}+1\right)} \sqrt{L_{2}\left(L_{2}+1\right)} \sqrt{\left[j, j_{a}\right]}\left[L_{1}, L_{2}, j_{\nu}\right]\left(-\lambda_{1}\right)^{p_{1}}\left(-\lambda_{2}\right)^{p_{2}} \\
& \quad \times \sum_{\kappa m_{j} m_{l}} e^{i \Delta_{j l} l}(-i)^{l} Y_{l m_{l}}\left(\hat{\boldsymbol{p}}_{e}\right)(-1)^{2 j-m_{j}+2 j_{\nu}-m_{\nu}+1} \\
& \left\langle l m_{l}, 1 / 2 m_{e} \mid j m_{j}\right\rangle\left\langle j m_{j}, L_{2}-\lambda_{2} \mid j_{\nu} m_{\nu}\right\rangle \\
& \quad \times \sum_{m_{a}}\left\langle j_{\nu} m_{\nu}, L_{1}-\lambda_{1} \mid j_{a} m_{a}\right\rangle\left\langle j_{a} \frac{1}{2}, L_{2} 0 \mid j_{\nu}-\frac{1}{2}\right\rangle \\
& \left\langle l_{\nu} \frac{1}{2}, L_{1} 0 \mid j-\frac{1}{2}\right\rangle \prod_{l_{a}, L_{1}, l_{\nu}, p_{1}} \prod_{l_{\nu}, L_{2}, l, p_{2}} U_{\kappa_{a} \kappa}^{p_{1} L_{\nu} p_{2} L_{2}}\left(\omega_{1}, \omega_{2}\right)
\end{aligned}
$$

where $j_{\nu}, l_{\nu}$ are total and orbital angular momentum quantum numbers of intermediate virtual state, respectively. The function $[L]=2 L+1$, and $\prod$ are defined as $\prod_{l_{1}, l_{2}, l_{3}, p}=1$, if the sum $l_{1}+l_{2}+l_{3}+p$ is odd, and $\prod_{l_{1}, l_{2}, l_{3}, p}=0$ otherwise.

The electron wave function in the radial transition amplitude $U_{\kappa_{\alpha} \kappa_{\nu} \kappa}^{p L p^{\prime} L^{\prime}}$ was obtained using six different screening potentials (core-Hartree, Salvat, Kohn-Sham, Local-Dirac-Fock, Perdew-Zunger, and Slater potentials). All screening potentials predict similar energy sharing dependence of the total TPI cross section, we choose the results presented in Core-Hartree screening potential. The sum in transition amplitude (5) runs over all multipole orders of the electron-photon inter- action operator and over the complete energy spectrum of the intermediate virtual states. However, we employ the dipole approximation and limit the maximum value $L_{\max }=1$ of the multipoles for each photon. This numerical limitation justifies the sufficient convergence of the total cross section less than $0.1 \%$. The infinite summation over the intermediate virtual states can be reduced to a finite sum over a pseudo-spectrum by employing a finite basis set constructed from B splines [36].

In the next subsection, we investigate the dependence of total TPI cross section on the polarization of the two incident photons and hence derive the corresponding expressions of the total cross section.

\subsection{Density matrix and total cross section}

Here, we use the photon helicity density matrix in order to characterize the polarization of each incident photon. The helicity density matrix for the first photon can be conveniently expressed in terms of Stokes parameter for any degree of linear $\left(P_{\gamma}^{l}=\sqrt{P_{1}^{2}+P_{2}^{2}}\right)$ and circular $\left(P_{\gamma}^{c}=P_{3}\right)$ polarization $[37]$

$$
\left\langle\boldsymbol{k} \lambda_{1}\left|\hat{\rho}_{\gamma_{1}}\right| \boldsymbol{k} \lambda_{1}^{\prime}\right\rangle=\frac{1}{2}\left(\begin{array}{cc}
1+P_{\gamma_{1}}^{c} & P_{\gamma_{1}}^{l} \\
P_{\gamma_{1}}^{l} & 1-P_{\gamma_{1}}^{c}
\end{array}\right) .
$$

As for two linearly polarized photons, it is practical to consider a polarization angle $\psi$ between these two copropagating photons, which are typically used in TPI experiments. We can write the helicity density matrix for the second photon in the following way

$$
\left\langle\boldsymbol{k} \lambda_{2}\left|\hat{\rho}_{\gamma_{2}}\right| \boldsymbol{k} \lambda_{2}^{\prime}\right\rangle=\frac{1}{2}\left(\begin{array}{cc}
1+P_{\gamma_{2}}^{c} & P_{\gamma_{2}}^{l} e^{-2 i \psi} \\
P_{\gamma_{2}}^{l} e^{2 i \psi} & 1-P_{\gamma_{2}}^{c}
\end{array}\right) .
$$

The corresponding total cross section of two-color twophoton $K$-shell ionization can be expressed by

$$
\begin{aligned}
\sigma= & \frac{8 \pi^{3} \alpha^{2}}{\omega_{1} \omega_{2}} \sum_{\lambda_{1} \lambda_{2} \lambda_{1}^{\prime} \lambda_{2}^{\prime}}\left\langle\boldsymbol{k} \lambda_{1}\left|\hat{\rho}_{\gamma_{1}}\right| \boldsymbol{k} \lambda_{1}^{\prime}\right\rangle\left\langle\boldsymbol{k} \lambda_{2}\left|\hat{\rho}_{\gamma_{2}}\right| \boldsymbol{k} \lambda_{2}^{\prime}\right\rangle \\
& \times \int d \hat{\boldsymbol{p}}_{e} \sum_{m_{e}} M_{p_{e} m_{e} \lambda_{2}}^{\lambda_{1} \lambda_{2}} M_{\boldsymbol{p}_{e} m_{e}}^{\lambda_{1}^{\prime} \lambda_{2}^{\prime} *} .
\end{aligned}
$$

This second-order total cross section has the units of $\left[L^{4} T\right]$.

Taking into account the two independent polarized photons, we can obtain the expansion of total cross section within relativistic framework for two-color twophoton $K$-shell ionization. Herein, we employ the electric dipole approximation, in which the radial transition amplitude becomes $U_{\kappa_{a} \kappa_{\nu} \kappa}=U_{\kappa_{a} \kappa_{\nu} \kappa}^{E 1 E 1}\left(\omega_{1}, \omega_{2}\right)+$ $U_{\kappa_{a} \kappa_{\nu} \kappa}^{E 1 E 1}\left(\omega_{2}, \omega_{1}\right)$, where $p$ and $L$ equal to 1 . In the case of TPI of $K$-shell electron, according to the selection rule, the Dirac quantum number of intermediate virtual state can take the values $\kappa_{\nu}=(-2,1)$, and of photoelectron state take the values $\kappa=(-3,-1,2)$. With this in mind, the expression (8) can be presented in terms of radial transition amplitudes as well as Stokes parameters 


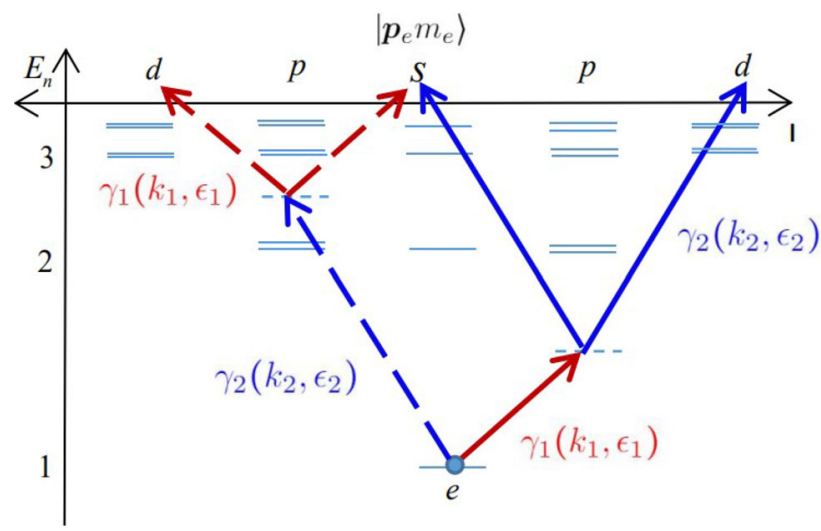

Fig. 1 The electric dipole transitions of two-color twophoton $K$-shell ionization. The horizontal axis represents the orbital angular momentum of the active electron, and the vertical axis $E_{n}$ represents the energy of the electron shells. In the nonrelativistic limit, $s \rightarrow p \rightarrow s$ and $s \rightarrow p \rightarrow d$ channels are opened to the TPI process for each incident photon. Thereby four transition paths exist totally. The full lines show the process that the first photon with $\gamma_{1}$ excites the initial state into an intermediate virtual state, and the second photon with $\gamma_{2}$ ionizes the electron from an intermediate virtual state into a continuum state. The dashed lines indicate the interchange of the interaction sequence of the two photons with the atom, the first and the second photon are absorbed with $\gamma_{2}$ and $\gamma_{1}$, respectively

$$
\begin{aligned}
\sigma^{(r)} & =\frac{2 \pi^{3} \alpha^{2}}{25 \omega_{1} \omega_{2}}\left[50\left(1-P_{\gamma_{1}}^{c} P_{\gamma_{2}}^{c}\right) U_{-11-1}^{2}+25\left[3 P_{\gamma_{1}}^{l} P_{\gamma_{2}}^{l} \cos 2 \psi\right.\right. \\
& \left.+5\left(1-P_{\gamma_{1}}^{c} P_{\gamma_{2}}^{c}\right)\right] U_{-1-2-1}^{2}+25\left[6 P_{\gamma_{1}}^{l} P_{\gamma_{2}}^{l} \cos 2 \psi\right. \\
& \left.+2\left(1-P_{\gamma_{1}}^{c} P_{\gamma_{2}}^{c}\right)\right] U_{-11-1} U_{-1-2-1} \\
& +50\left(2+P_{\gamma_{1}}^{c} P_{\gamma_{2}}^{c}\right) U_{-112}^{2} \\
& +2\left(8-5 P_{\gamma_{1}}^{c} P_{\gamma_{2}}^{c}-6 P_{\gamma_{1}}^{l} P_{\gamma_{2}}^{l} \cos 2 \psi\right) U_{-1-22}^{2} \\
& +10\left(1+5 P_{\gamma_{1}}^{c} P_{\gamma_{2}}^{c}+3 P_{\gamma_{1}}^{l} P_{\gamma_{2}}^{l} \cos 2 \psi\right) U_{-112} U_{-1-22} \\
& \left.+27\left(7+5 P_{\gamma_{1}}^{c} P_{\gamma_{2}}^{c}+P_{\gamma_{1}}^{l} P_{\gamma_{2}}^{l} \cos 2 \psi\right) U_{-1-2-3}^{2}\right]
\end{aligned}
$$

As we can see from Eq. (9), the relativistic total cross section depends on five radial transition amplitudes $\left(U_{-11-1}, U_{-1-2-1}, U_{-112} U_{-1-22}, U_{-1-2-3}\right)$, which are all real numbers in our calculation.

However, a nonrelativistic description is completely sufficient for many atoms and ions, due to the negligible relativistic effects [38]. The TPI process has been already studied within nonrelativistic framework, and the relativistic corrections to the total cross section have been evaluated in Ref. [19]. In the nonrelativistic limit, the transition amplitudes as well as phase factors remain unaffected by the fine structure splitting, we can neglect the splitting effect and approximate $U_{-11-1}=U_{-1-2-1}=U_{s}, \quad U_{-112}=U_{-1-22}=U_{-1-2-3}=U_{d}$. Thus, the five relativistic paths for each photon reduce to two nonrelativistic paths. By performing the simplification, the nonrelativistic expression of total cross section reads as

$$
\begin{aligned}
\sigma^{(n r)}= & \frac{18 \pi^{3} \alpha^{2}}{5 \omega_{1} \omega_{2}}\left[5\left(1+P_{\gamma_{1}}^{l} P_{\gamma_{2}}^{l} \cos 2 \psi-P_{\gamma_{1}}^{c} P_{\gamma_{2}}^{c}\right) U_{s}^{2}(10)\right. \\
& \left.+\left(7+P_{\gamma_{1}}^{l} P_{\gamma_{2}}^{l} \cos 2 \psi+5 P_{\gamma_{1}}^{c} P_{\gamma_{2}}^{c}\right) U_{d}^{2}\right] .
\end{aligned}
$$

This total cross section is also consistent with the theoretical description of two-photon two-color abovethreshold ionization in Ref. [14]. In contrast, our expression (10) makes it easier to explain the dependence of the TPI on the radial transition amplitudes $U_{\kappa_{\alpha} \kappa_{\nu} \kappa}$ as well as the polarization angle $\psi$. In the following subsection, we will discuss the cross section ratio of different polarization combinations of the two incident photons, in order to study the polarization dependence of the two-photon ionization.

\subsection{Cross section ratios}

The first step of deriving the cross section ratio is to set a reference total cross section as a denominator. As we already mentioned in Sect. 2.2, two linearly polarized photons are typically used in TPI experiments; therefore, we take into account the ionization with two parallel linearly polarized photons both along the $\mathrm{x}$ axis, in which the Stokes parameters take the values $P_{\gamma_{1}}^{l}=P_{\gamma_{2}}^{l}=1, P_{\gamma_{1}}^{c}=P_{\gamma_{2}}^{c}=0$, the polarization angle $\psi=0$, and obtain the total cross section $\sigma_{x x}^{(n r)}$. We construct the cross section ratios of four polarization combinations

$$
\begin{aligned}
& \frac{\sigma_{x y}^{(n r)}}{\sigma_{x x}^{(n r)}}=\frac{3 R}{5+4 R}, \\
& \frac{\sigma_{++}^{(n r)}}{\sigma_{x x}^{(n r)}}=\frac{6 R}{5+4 R}, \\
& \frac{\sigma_{+-}^{(n r)}}{\sigma_{x x}^{(n r)}}=1-\frac{3 R}{5+4 R}, \\
& \frac{\sigma_{x+}^{(n r)}}{\sigma_{x x}^{(n r)}}=\frac{1}{2}+\frac{3 R}{10+8 R},
\end{aligned}
$$

where we use the notation $R=U_{d}^{2} / U_{s}^{2}$. The subscripts $\mathrm{x}$ and $\mathrm{y}$ represent the linear polarization direction, while \pm stands for circular polarization with the helicities \pm 1 . For instance, the notation $\sigma_{++}^{(n r)}$ represents the total TPI cross section of two fully circularly polarized photons with helicity +1 . The corresponding Stokes parameters are $P_{\gamma_{1}}^{l}=P_{\gamma_{2}}^{l}=0, P_{\gamma_{1}}^{c}=P_{\gamma_{2}}^{c}=1$.

\section{Results and discussion}

In Sect. 2.1, we obtained the general expression (trans) of transition amplitude for two-color two-photon $K$ shell ionization process. In Sect. 2.2, the total cross section (8) has been derived within the relativistic 
and nonrelativistic framework. Finally, in Sect. 2.3, the cross section ratios of different polarization combinations have been derived. In this section, we present the numerical calculation of the total cross section and cross section ratios within the relativistic framework. We selected Ne and Ge as our target atoms, which have been already studied in experiments $[7,8]$. Figure total cross section shows the dependence of the relativistic total cross section of two-color two-photon $K$-shell ionization on the energy sharing between the two incident photons, $\xi=\frac{\omega_{1}}{\omega_{1}+\omega_{2}}$, calculated for Ne and Ge atoms.

We also investigated the dependence of the total cross section on combined excess energy, which represents the ratio of sum energies of the two incident photons to the ionization threshold energy of $K$-shell electron, $\varepsilon=\hbar\left(\omega_{1}+\omega_{2}\right) / E_{1}$. Higher values of combined excess energy have not been considered for the study to avoid various resonances in TPI. The presented energy ranges were chosen from the TPI threshold up to $1 s \rightarrow 2 p$ resonance transition energy. In particular, as we can see from these figures, the total cross section reaches the minimum value for all three cases of excess energy, especially if the energy sharing $\xi$ reaches 0.5 , which indicates the energies of two photons are identical.

Moreover, if one of the photon energies $\omega_{1}$ or $\omega_{2}$ reaches the energy difference between the $1 \mathrm{~s}$ and $2 \mathrm{p}$ states of the electron transition, $\Delta E_{1 s \rightarrow 2 p}=E_{1}-E_{2}$, the symmetric resonance peaks appear as shown in Fig. 2. In the region between these two resonance peaks, we also observe that the width becomes narrower with higher $\varepsilon$. We derived a formula for the width $d$ between two resonance peaks as a function of $\Delta E_{1 s \rightarrow 2 p}$ as well as $\varepsilon$

$$
d=\frac{2}{\varepsilon} \frac{\Delta E_{1 s \rightarrow 2 p}}{E_{1}}-1,
$$

from this equation, follows that the width $d$ is proportional to $1 / \varepsilon$ as shown in Fig. 2. Moreover, if we compare the results of $\mathrm{Ne}$ and $\mathrm{Ge}$ for equal $\varepsilon$, we note that the width $d$ depends also on the energy percentage $\Delta E_{1 s \rightarrow 2 p} / E_{1}$, which is determined by atom nuclear charge.

Next, we investigated the cross section ratios of $\mathrm{Ne}$ and Ge for four different polarization combinations of the two incident photons. Figure 3 shows the relativistic calculation of the cross section ratios for different polarization combinations, implying a similar energy sharing dependence for different combined excess energy as well as different atoms. The values of the ratio reach either minimum or maximum if the energy sharing is equal to 0.5. Moreover, we found that in the cases of $\sigma_{x y}^{(n r)} / \sigma_{x x}^{(n r)}$, $\sigma_{++}^{(n r)} / \sigma_{x x}^{(n r)}$ and $\sigma_{x+}^{(n r)} / \sigma_{x x}^{(n r)}$, the closer the energy sharing $\xi$ near the center, the smaller the cross section ratio is, while the contrary energy sharing dependence of $\sigma_{+-}^{(n r)} / \sigma_{x x}^{(n r)}$ can be distinguished from above three cases.

In the dipole approximation, the transition allows two possible ionization channels in the TPI process: $s \rightarrow p \rightarrow s$ and $s \rightarrow p \rightarrow d$. We can see that $U_{s}$ van-
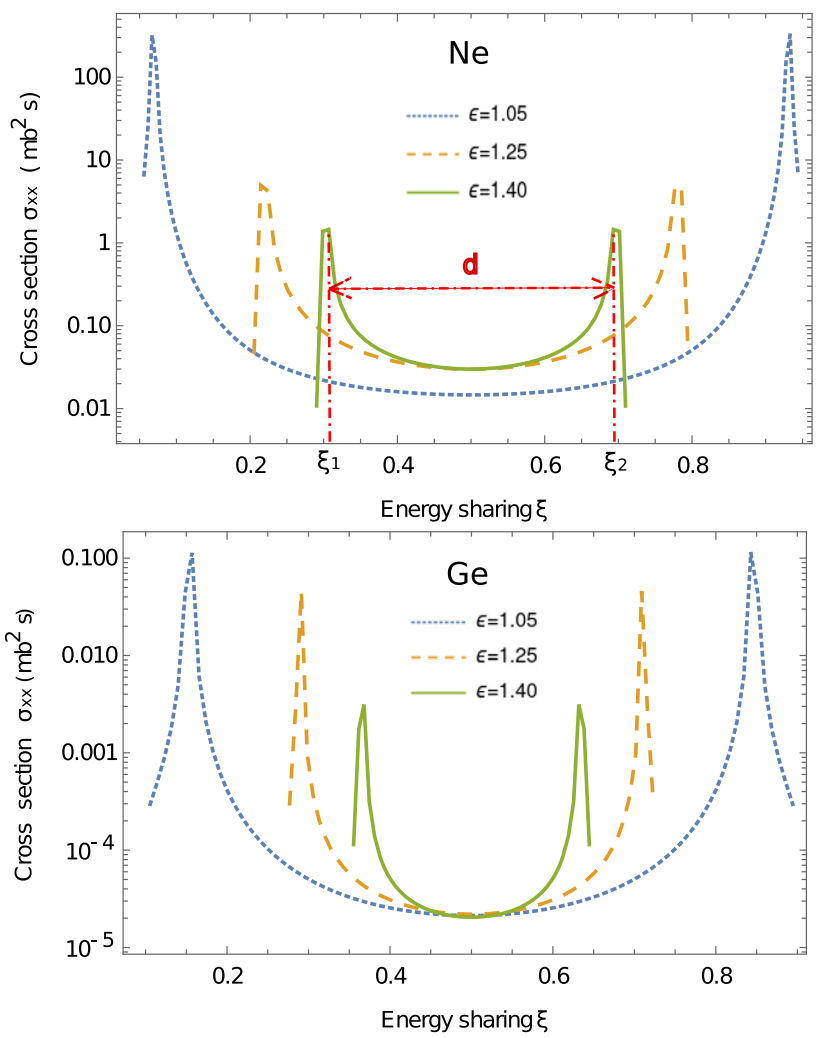

Fig. 2 The total cross section for ionization of $\mathrm{Ne}$ and $\mathrm{Ge}$ by two linearly polarized photons as a function of energy sharing $\xi$. The energy sharing $\xi=\frac{\omega_{1}}{\omega_{1}+\omega_{2}}$ implies the energy distribution between the two incident photons. The combined excess energy $\varepsilon=\frac{\hbar\left(\omega_{1}+\omega_{2}\right)}{E_{1}}$ represents the total energy carried by the two photons normalized to the binding energy of the $1 \mathrm{~s}$ electron. The short dashed line corresponds to $\varepsilon=1.05$, the long dashed line corresponds to $\varepsilon=1.25$ and solid line corresponds to $\varepsilon=1.40$

ishes in the numerator in Eqs.(11) and (2), indicating that the $s \rightarrow p \rightarrow s$ channel is closed for TPI with two co-rotating circularly polarized or two orthogonal linearly polarized photons. The boundary values of cross section ratio for each polarization combinations can be approximately found from Fig. $3\left(\sigma_{x y}^{(n r)} / \sigma_{x x}^{(n r)} \cong 0.75\right.$, $\sigma_{++}^{(n r)} / \sigma_{x x}^{(n r)} \cong 1.5, \sigma_{+-}^{(n r)} / \sigma_{x x}^{(n r)} \cong 0.25, \sigma_{x y}^{(n r)} / \sigma_{x x}^{(n r)} \cong$ 0.875 ) and are well predicted by Eqs. (11-14) under the circumstance if $R \gg 1$. In particular, the stable convergence around $3 / 2$ of known estimate $\sigma_{++}^{(n r)} / \sigma_{x x}^{(n r)}$ for TPI process is also in agreement with the theoretical description mentioned in Ref. [39]. As the combined excess energy increases from 1.05 to 1.40 , the cross section ratios approach more smoothly to their boundary values. These boundary values showed the dominance of $s \rightarrow p \rightarrow d$ channel, which can verify the Fano's propensity rule discussed in Ref. [40].

From Fig. 3, by comparing the deviations of cross section ratios for $\mathrm{Ne}$ and $\mathrm{Ge}$ atoms, we observed the stronger screening effect occurring in the Ne atom. The screening effect is a result of interelectronic interaction in the electron shells and leads to the decrease of the 
$\mathrm{Ne}$
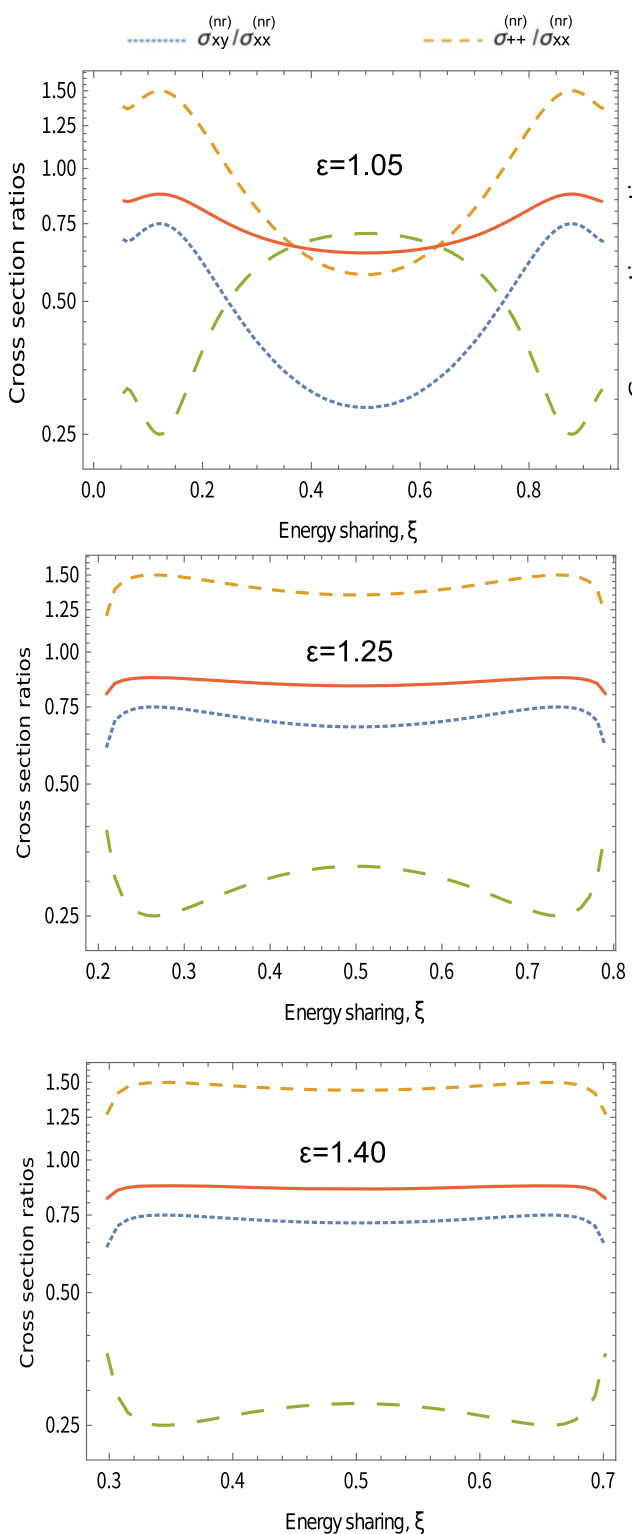

$\mathrm{Ge}$
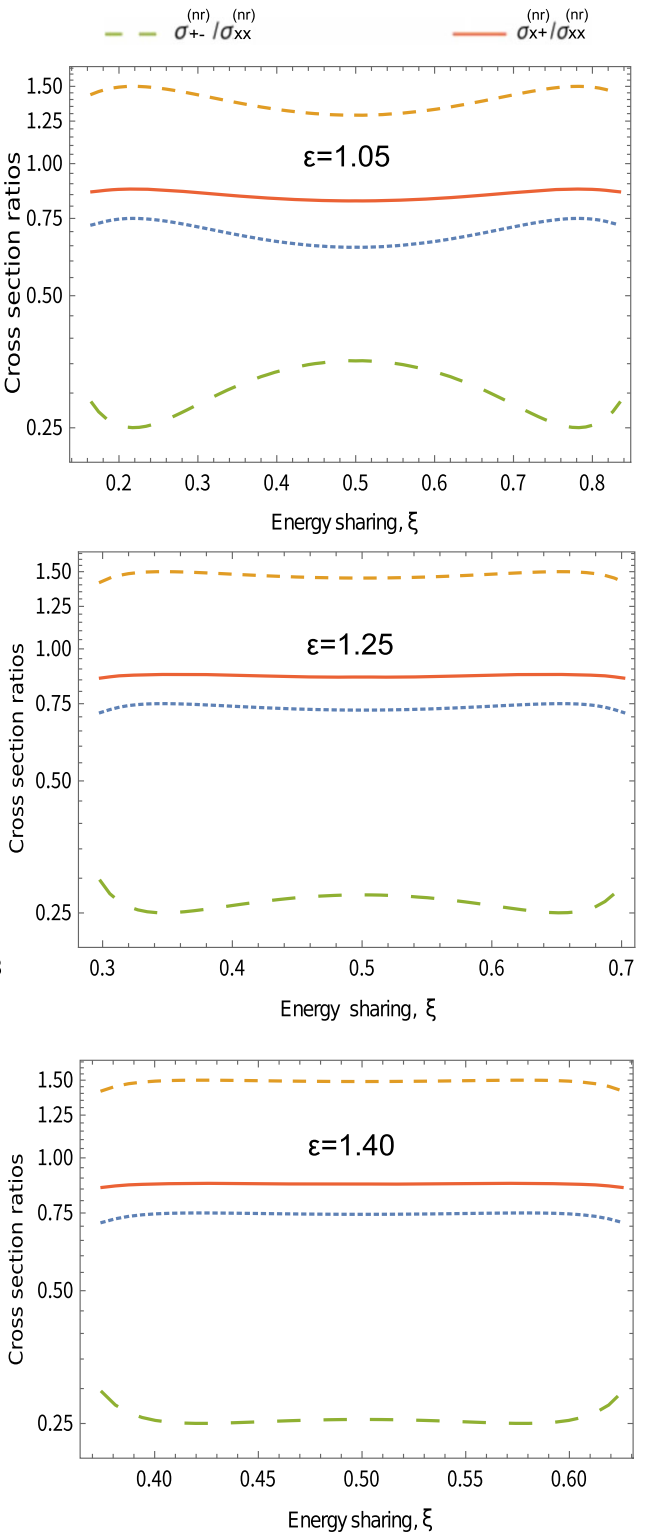

Fig. 3 The cross section ratios of bichromatic two-photon $K$-shell ionization, where $x$ represents the linearly polarization direction along $x$-axis, the corresponding angle $\psi=0$ and Stokes parameters $P_{\gamma}^{l}=1, P_{\gamma}^{c}=1 ; y$ represents the linearly polarization direction along $y$-axis, the corresponding angle $\psi=\pi / 2$ and Stokes parameter $P_{\gamma}^{l}=1, P_{\gamma}^{c}=1$. The notation \pm stands for the helicity \pm 1 of the circular polarization (the spin projection onto $\breve{k}$ direction), the corresponding Stokes parameters $P_{\gamma}^{l}=0, P_{\gamma}^{c}= \pm 1$

electron binding energies. In contrast to the Ge atom, the Ne atom has stronger interaction between the innershell electrons and electrons from other shells, which results in stronger deviations for all cross section ratios with three excess energies. Furthermore, due to the strong drop of the dominant $s \rightarrow p \rightarrow d$ channel near the ionization threshold, a magnification of the screening effects can be also observed for $\varepsilon=1.05$ of Ne atom, where the strongest deviations of all cross section ratios for different polarization combinations occur, this effect is also in agreement with the partial-wave analysis of two-photon ionization in Ref. [18].

\section{Summary and outlook}

To conclude, We have studied the two-color two-photon $K$-shell ionization within relativistic second order perturbation theory and the independent particle approximation. The analytical expressions for the total cross section of the ionization process were obtained in terms 
of radial transition amplitudes and Stokes parameters. We also derived the cross section ratios of different combinations of the polarization state of the two incident photons within the nonrelativistic framework. Detailed calculations of the total cross section and cross section ratios for $\mathrm{Ne}$ and $\mathrm{Ge}$ atoms have been carried out. In the numerical results, we found that the total cross section depends on the energy sharing. The local minimum value occurs if the energies of the two photons are identical. Besides, the dependence of two-color two-photon $K$-shell ionization on the polarization state of two incident photons has also been clarified in this paper. The boundary values of cross section ratios have been predicted for each polarization combination with the analytical expressions and were well confirmed by the numerical computation results. Moreover, a strong deviation of cross section ratios near the TPI threshold of the Ne atom was observed, which relates to the strong screening effect. These insights are helpful to further our understanding of the two-photon ionization process.

Acknowledgements This work was supported by the Ministry of Science and Education of the Russian Federation (Project No. 075-15-2021-1349). J.F also acknowledges the general help of Baghdasar Baghdasaryan.

\section{Author contributions}

JF, JH and AVV conceived the original idea. JF and JH developed the theoretical formalism, JF performed both the analytical calculations and numerical simulations with support from JH and AVV. SF helped supervise the findings of this work. All authors discussed the results and contributed to the final manuscript.

Funding Information Open Access funding enabled and organized by Projekt DEAL.

Data Availability Statement This manuscript has no associated data or the data will not be deposited. [Authors' comment: In this paper, we use programming to implement numerical simulations and present the trends of the relevant parameters in form of figures. Moreover, the results of the study were analysed from a large number of data calculated by the codes we programmed which is not the central focus of this paper.]

Open Access This article is licensed under a Creative Commons Attribution 4.0 International License, which permits use, sharing, adaptation, distribution and reproduction in any medium or format, as long as you give appropriate credit to the original author(s) and the source, provide a link to the Creative Commons licence, and indicate if changes were made. The images or other third party material in this article are included in the article's Creative Commons licence, unless indicated otherwise in a credit line to the material. If material is not included in the article's Creative Commons licence and your intended use is not permitted by statu- tory regulation or exceeds the permitted use, you will need to obtain permission directly from the copyright holder. To view a copy of this licence, visit http://creativecomm ons.org/licenses/by/4.0/.

\section{References}

1. V. Richardson, J.T. Costello, D. Cubaynes, S. Düsterer, J. Feldhaus, H.W. van der Hart, P. Juranić, W.B. Li, M. Meyer, M. Richter, A.A. Sorokin, K. Tiedke, Phys. Rev. Lett. 105, 013001 (2010). https://doi.org/10.1103/ PhysRevLett.105.013001

2. L. Young, E. Kanter, B. Krässig, Y. Li, A. March, T. Pratt, R. Santra, S. Southworth, N. Rohringer, L. Dimauro, G. Doumy, C. Roedig, N. Berrah, L. Fang, M. Hoener, P. Bucksbaum, J. Cryan, S. Ghimire, J. Glownia, M. Messerschmidt, Nature 466, 56 (2010). https:// doi.org/10.1038/nature09177

3. C. Callegari, A.N. Grum-Grzhimailo, K.L. Ishikawa, K.C. Prince, G. Sansone, K. Ueda (2020), arXiv:2008.11024 [physics.atom-ph]

4. P. Emma, R. Akre, J. Arthur, R. Bionta, C. Bostedt, J. Bozek, A. Brachmann, P. Bucksbaum, R. Coffee, F.-J. Decker, Y. Ding, D. Dowell, S. Edstrom, A. Fisher, J. Frisch, S. Gilevich, J. Hastings, G. Hays, P. Hering, J. Galayda, Nat. Photonics 4, 641 (2010). https://doi.org/ 10.1038/nphoton.2010.176

5. T. Ishikawa, H. Aoyagi, T. Asaka, Y. Asano, N. Azumi, T. Bizen, H. Ego, K. Fukami, T. Fukui, Y. Furukawa, S. Goto, H. Hanaki, T. Hara, T. Hasegawa, T. Hatsui, A. Higashiya, T. Hirono, N. Hosoda, M. Ishii, N. Kumagai, Nat. Photonics 6, 540 (2012). https://doi.org/10.1038/ nphoton.2012.141

6. J. Amann, W. Berg, V. Blank, F.-J. Decker, Y. Ding, P. Emma, Y. Feng, J. Frisch, D. Fritz, J. Hastings, Z. Huang, J. Krzywinski, R. Lindberg, H. Loos, A. Lutman, H.-D. Nuhn, D. Ratner, J. Rzepiela, D. Shu, D. Zhu, Nat. Photonics 6, 693 (2012). https://doi.org/10. 1038/nphoton.2012.180

7. G. Doumy, C. Roedig, S.-K. Son, C.I. Blaga, A.D. DiChiara, R. Santra, N. Berrah, C. Bostedt, J.D. Bozek, P.H. Bucksbaum, J.P. Cryan, L. Fang, S. Ghimire, J.M. Glownia, M. Hoener, E.P. Kanter, B. Krässig, M. Kuebel, M. Messerschmidt, G.G. Paulus, D.A. Reis, N. Rohringer, L. Young, P. Agostini, L.F. DiMauro, Phys. Rev. Lett. 106, 083002 (2011). https://doi.org/10.1103/ PhysRevLett.106.083002

8. K. Tamasaku, E. Shigemasa, Y. Inubushi, T. Katayama, K. Sawada, H. Yumoto, H. Ohashi, H. Mimura, M. Yabashi, K. Yamauchi, T. Ishikawa, Nat. Photonics 8, 313 (2014). https://doi.org/10.1038/nphoton.2014.10

9. S. Ghimire, M. Fuchs, J. Hastings, S.C. Herrmann, Y. Inubushi, J. Pines, S. Shwartz, M. Yabashi, D.A. Reis, Phys. Rev. A 94, 043418 (2016). https://doi.org/10. 1103/PhysRevA.94.043418

10. J. Szlachetko, J. Hoszowska, J.-C. Dousse, M. Nachtegaal, W. Błachucki, Y. Kayser, J. Sa, M. Messerschmidt, S. Boutet, G. Williams, C. David, G. Smolentsev, J. Bokhoven, B. Patterson, T. Penfold, G. Knopp, M. 
Pajek, R. Abela, C. Milne, Sci. Rep. 6, 33292 (2016). https://doi.org/10.1038/srep33292

11. K. Tamasaku, E. Shigemasa, Y. Inubushi, I. Inoue, T. Osaka, T. Katayama, M. Yabashi, A. Koide, T. Yokoyama, T. Ishikawa, Phys. Rev. Lett. 121, 083901 (2018). https://doi.org/10.1103/PhysRevLett. 121.083901

12. S. Novikov, A. Hopersky, J. Phys. B 33, 2287 (2000). https://doi.org/10.1088/0953-4075/33/12/310

13. A. Sytcheva, S. Pabst, S.-K. Son, R. Santra, Phys. Rev. A 85, 023414 (2012). https://doi.org/10.1103/ PhysRevA.85.023414

14. A.N. Grum-Grzhimailo, E.V. Gryzlova, Phys. Rev. A 89, 043424 (2014). https://doi.org/10.1103/PhysRevA. 89.043424

15. D.I.R. Boll, O.A. Fojón, C.W. McCurdy, A. Palacios, Phys. Rev. A 99, 023416 (2019). https://doi.org/10. 1103/PhysRevA.99.023416

16. P. Koval, S. Fritzsche, A. Surzhykov, J. Phys. B 36, 873 (2003). https://doi.org/10.1088/0953-4075/36/5/307

17. P. Koval, Two-photon ionization of atomic inner-shells, Ph.D. thesis, University of Kassel (2004)

18. J. Hofbrucker, A.V. Volotka, S. Fritzsche, Phys. Rev. A 94, 063412 (2016). https://doi.org/10.1103/PhysRevA. 94.063412

19. J. Hofbrucker, A.V. Volotka, S. Fritzsche, Phys. Rev. A 96, 013409 (2017). https://doi.org/10.1103/PhysRevA. 96.013409

20. A.A. Lutman, R. Coffee, Y. Ding, Z. Huang, J. Krzywinski, T. Maxwell, M. Messerschmidt, H.-D. Nuhn, Phys. Rev. Lett. 110, 134801 (2013). https://doi.org/10.1103/ PhysRevLett.110.134801

21. T. Hara, Y. Inubushi, T. Katayama, T. Sato, H. Tanaka, T. Tanaka, T. Togashi, K. Togawa, K. Tono, M. Yabashi, T. Ishikawa, Nat. Commun. 4, 2919 (2013). https://doi.org/10.1038/ncomms3919

22. A.A. Lutman, F.-J. Decker, J. Arthur, M. Chollet, Y. Feng, J. Hastings, Z. Huang, H. Lemke, H.-D. Nuhn, A. Marinelli, J.L. Turner, S. Wakatsuki, J. Welch, D. Zhu, Phys. Rev. Lett. 113, 254801 (2014). https://doi.org/ 10.1103/PhysRevLett.113.254801

23. A. Saá Hernández, E. Prat, Phys. Rev. Accel. Beams 22, 030702 (2019). https://doi.org/10.1103/ PhysRevAccelBeams.22.030702

24. P. Dijkstal, A. Malyzhenkov, S. Reiche, E. Prat, Phys. Rev. Accel. Beams 23, 030703 (2020). https://doi.org/ 10.1103/PhysRevAccelBeams.23.030703

25. I. Inoue, T. Osaka, T. Hara, M. Yabashi, J. Synchrotron Radiat. 27, 1720 (2020). https://doi.org/10. 1107/S1600577520011716

26. K.-J. Kim, Nucl. Instrum. Methods Phys. Res. A 445, 329 (2000). https://doi.org/10.1016/ S0168-9002(00)00137-6

27. Y. Li, B. Faatz, J. Pflueger, E. Saldin, E. Schneidmiller, M. Yurkov, 30th International Free Electron Laser Conference, FEL 2008 (2008)

28. Y. Ding, Z. Huang, Phys. Rev. ST Accel. Beams 11, 030702 (2008). https://doi.org/10.1103/PhysRevSTAB. 11.030702

29. T. Zhang, H.-X. Deng, W.-Q. Zhang, G.-R. Wu, D.-X. Dai, D. Wang, X.-M. Yang, Z.-T. Zhao, Chin. Phys. C 37, 118101 (2013). https://doi.org/10.1088/1674-1137/ $37 / 11 / 118101$
30. E.A. Schneidmiller, M.V. Yurkov, Phys. Rev. ST Accel. Beams 16, 110702 (2013). https://doi.org/10.1103/ PhysRevSTAB.16.110702

31. E. Ferrari, E. Roussel, J. Buck, C. Callegari, R. Cucini, G. De Ninno, B. Diviacco, D. Gauthier, L. Giannessi, L. Glaser, G. Hartmann, G. Penco, F. Scholz, J. Seltmann, I. Shevchuk, J. Viefhaus, M. Zangrando, E.M. Allaria, Phys. Rev. Accel. Beams 22, 080701 (2019). https:// doi.org/10.1103/PhysRevAccelBeams.22.080701

32. N. Sudar, R. Coffee, E. Hemsing, Phys. Rev. Accel. Beams 23, 120701 (2020). https://doi.org/10.1103/ PhysRevAccelBeams.23.120701

33. D.A. Varshalovich, A.N. Moskalev, V.K. Khersonskii, Quantum Theory of Angular Momentum (World Scientific, Singapore, 1988). https://doi.org/10.1142/0270

34. J. Eichler, T. Stöhlker, Phys. Rep. 439, 1 (2007). https://doi.org/10.1016/j.physrep.2006.11.003

35. W. Johnson, Atomic Structure Theory: Lectures on Atomic Physics (Springer, Berlin, 2007), p. 1. https:// doi.org/10.1007/978-3-540-68013-0

36. J. Sapirstein, W.R. Johnson, J. Phys. B 29, 5213 (1996). https://doi.org/10.1088/0953-4075/29/22/005

37. K. Blum, Density Matrix Theory and Applications, 3rd edn. (Springer, Berlin, 2012). https://doi.org/10.1007/ 978-3-642-20561-3

38. N. Manakov, S. Marmo, S. Sviridov, J. Exp, Theor. Phys. 108, 557 (2009). https://doi.org/10.1134/ S1063776109040025

39. P. Lambropoulos, Phys. Rev. Lett. 28, 585 (1972). https://doi.org/10.1103/PhysRevLett.28.585

40. U. Fano, Phys. Rev. A 32, 617 (1985). https://doi.org/ 10.1103/PhysRevA.32.617 